\title{
Comparison of minimally invasive surgical approaches for hysterectomy at a community hospital: robotic-assisted laparoscopic hysterectomy, laparoscopic-assisted vaginal hysterectomy and laparoscopic supracervical hysterectomy
}

\author{
Bang N. Giep $\cdot$ Hoang N. Giep $\cdot$ Helen B. Hubert
}

Received: 27 April 2010/Accepted: 29 June 2010/Published online: 10 August 2010

(C) The Author(s) 2010. This article is published with open access at Springerlink.com

\begin{abstract}
The study reported here compares outcomes of three approaches to minimally invasive hysterectomy for benign indications, namely, robotic-assisted laparoscopic (RALH), laparoscopic-assisted vaginal (LAVH) and laparoscopic supracervical (LSH) hysterectomy. The total patient cohort comprised the first 237 patients undergoing robotic surgeries at our hospital between August 2007 and June 2009; the last 100 patients undergoing LAVH by the same surgeons between July 2006 and February 2008 and 165 patients undergoing LAVHs performed by nine surgeons between January 2008 and June 2009; 87 patients undergoing LSH by the same nine surgeons between January 2008 and June 2009. Among the RALH patients were cases of greater complexity: (1) higher prevalence of prior abdominopelvic surgery than that found among LAVH patients; (2) an increased number of procedures for endometriosis and pelvic reconstruction. Uterine weights also were greater in RALH patients [207.4 vs. 149.6 (LAVH; $P<0.001)$ and $141.1 \mathrm{~g}(\mathrm{LSH} ; \quad P=0.005)$ ]. Despite case complexity, operative time was significantly lower in RALH than in LAVH (89.9 vs. $124.8 \mathrm{~min}$, $P<0.001)$ and similar to that in LSH $(89.6 \mathrm{~min})$. Estimated blood loss was greater in LAVH $(167.9 \mathrm{ml})$ than in
\end{abstract}

B. N. Giep · H. N. Giep

Department of Obstetrics and Gynecology, Spartanburg

Regional Medical Center, Spartanburg, SC, USA

H. B. Hubert

Department of Medicine, Stanford University School

of Medicine, Stanford, CA, USA

B. N. Giep $(\bowtie)$

Spartanburg and Pelham P.A., 250 North Grove Medical Park

Drive, Spartanburg, SC 29303, USA

e-mail: bgobgyn@yahoo.com
RALH (59.0 ml, $P<0.001)$ or LSH (65.7 ml, $P<0.001)$. Length of hospital stay was shorter for RALH than for LAVH or LSH. Conversion and complication rates were low and similar across procedures. Multivariable regression indicated that LAVH, obesity, uterine weight $\geq 250 \mathrm{~g}$ and older age predicted significantly longer operative time. The learning curve for RALH demonstrated improved operative time over the case series. Our findings show the benefits of RALH over LAVH. Outcomes in RALH can be as good as or better than those in LSH, suggesting the latter should be the choice primarily for women desiring cervixsparing surgery.

Keywords Robotics - Laparoscopy - Hysterectomy · Vaginal hysterectomy $\cdot$ Supracervical hysterectomy

\section{Introduction}

Although abdominal hysterectomy still accounts for approximately two-thirds of all benign hysterectomies performed in the USA [1, 2], minimally invasive techniques for this procedure have been slowly gaining acceptance. Recently published national data for 2005 indicate that vaginal hysterectomies were performed in about $22 \%$ of cases and that $14 \%$ of hysterectomies were done laparoscopically [2]. Studies have shown that minimally invasive techniques for benign hysterectomy are safe for the patient and result in decreased morbidity, shorter hospital stays, and a faster return to normal activity compared to open procedures [3, 4]. However, with conventional laparoscopic surgery, the surgeon generally experiences some decrease in visual acuity, lack of tactile sensory input, and, thus, greater difficulty in mastering the approach. Difficulty with hand-eye coordination with 
laparoscopy and the non-articulated instruments also makes this approach more difficult to master. These factors may explain a large part of the reluctance to adopt minimally invasive laparoscopic approaches over abdominal hysterectomy.

Improvements in minimally invasive techniques were introduced with 2005 Federal Drug Administration (FDA) approval of the da Vinci Surgical System (Intuitive Surgical, Sunnyvale, CA) for use in gynecologic procedures. Since that time, the use of the robotic system for hysterectomy has been shown to shorten the learning curve for laparoscopically naïve surgeons with improved threedimensional (3D) visual acuity, articulated wrist-like movement of instruments without tremor and ergonomic seating [5-7].

Few studies have compared clinical outcomes of the robot system with other minimally invasive techniques for hysterectomy. Payne and Dauterive demonstrated that patients who had undergone robotic-assisted compared to total laparoscopic hysterectomy had similar complication rates, but significantly less blood loss, fewer conversions to laparotomy and shorter hospital stays [7]. A recent investigation comparing robotic-assisted to traditional laparoscopic hysterectomy confirmed these findings [8]. A similar comparative study showed shorter hospital stays but longer operative times for patients who underwent a robotic procedure [9]. A number of studies have compared laparoscopic supracervical hysterectomy (LSH) to laparoscopic-assisted vaginal hysterectomy (LAVH) or to total laparoscopic hysterectomy. Results have repeatedly shown better outcomes with LSH than LAVH, i.e., shorter operative time, less blood loss and lower complication rates [10-12]. To date, however, there have been no comparative studies of robotic-assisted laparoscopic hysterectomy (RALH) to LAVH alone or to LSH. The goal of the study reported here was to provide such comparative data on clinical outcomes and learning curves for minimally invasive procedures in a community-practice setting.

\section{Materials and methods}

Patients and cases

The Spartanburg Regional Healthcare System is an integrated healthcare system delivering services, including ambulatory and inpatient surgery, to several counties in North and South Carolina. This study compares hysterectomies performed by multiple community surgeons at the Spartanburg Regional Medical Center, a facility within that system. Only two surgeons performed RALH during the time period of this study (BG, HG), so the robotic cohort ( $n=237)$ includes their entire consecutive RALH experience beginning with the first patient in August 2007 through to June 2009. Between July 2006 and February 2008, these same surgeons performed their last 100 LAVHs, transitioning almost exclusively to RALH in the latter half of 2007. Thus, the LAVH cohort for this study includes their last consecutive patients $(n=100)$ along with those of nine other gynecologists who performed LAVH at the same institution between January 2008 and June 2009 ( $n=165)$, for a total of 265 LAVH cases. In addition, consecutive LSH patients of the nine other gynecologists $(n=87)$ were identified during a similar time period, January 2008 to June 2009. Patients were selected for LSH, rather than LAVH, if they preferred a procedure that spared the cervix or if the surgeon believed it to be a better choice. There were too few total laparoscopic hysterectomies performed at the hospital during this time to form another comparison group. Every patient included in this study presented with a benign gynecologic condition.

\section{Surgical procedures}

All procedures were performed under general endotracheal anesthesia with preoperative antibiotics given. A steep Trendelenburg position was used with a gel pad underneath the patient for stabilization. A Foley catheter and uterine manipulator were placed for all surgeries.

\section{Robotic-assisted laparoscopic hysterectomy}

A $8.5-\mathrm{mm}$ trocar was placed at the umbilicus and 8-mm trocars were placed in the right and left lower quadrants. A 11-mm bladeless trocar was placed in the right upper quadrant. Docking of the robotic arms to the trocars followed by insertion of the 3-D camera was completed. A Gyrus PK Dissector (Gyrus ACMI, Maple Grove, MN) and monopolar scissors were inserted, at which time the console portion of the procedure began. Dissection of the bilateral round ligaments, adnexa and broad ligaments was performed in the standard manner. The bladder flap was created with a combination of sharp and blunt dissection. A colpotomy was performed with the monopolar scissors and carried circumferentially until the specimen was amputated from the vagina. The specimen was either removed vaginally or endoscopically, and the vaginal cuff was closed laparoscopically with interrupted figureof-eight stitches of 0 Vicryl.

\section{Laparoscopic-assisted vaginal hysterectomy}

To begin, the laparoscope was placed through a 5-mm umbilical trocar. Three additional $5-\mathrm{mm}$ ports were placed in the right and left lower quadrants and suprapubically. 
A Maryland bipolar dissector was used. Dissection of the bilateral round ligaments, adnexa, and broad ligaments was performed in the standard manner. The bladder flap was created with a combination of sharp and blunt dissection. A small anterior colpotomy was performed with monopolar scissors. The remainder of the procedure was completed vaginally. A posterior colpotomy was performed sharply, and then cardino-uterosacral ligaments and uterine vessels were divided and sutured. After the specimen was removed vaginally, the vaginal cuff was closed with 0 Vicryl in a running locked fashion.

\section{Laparoscopic supracervical hysterectomy}

A 5-mm trocar was placed at the umbilicus and two additional 5-mm trocars were placed in the right and left lower quadrants. Dissection of the bilateral round ligaments, adnexa and broad ligaments was performed in the standard manner using either Harmonic ACE curved shears (Ethicon, Cincinnati, $\mathrm{OH}$ ) or a LigaSure (Covidien, Mansfield, MA) device. The bladder was dissected free from the lower uterine segment, and the uterine vessels were coagulated and transected. The uterus was amputated from the cervix using either monopolar scissors or the Harmonic ACE curved shears and the endocervical canal fulgurated with the monopolar scissors. The specimen was then extracted with a Gynecare Morcellex Tissue Morcellator (Ethicon).

Patient characteristics and clinical procedures

The Institutional Review Board of Spartanburg Regional Hospital approved this study. A standardized retrospective chart review was completed by the research nursing staff, and quality control procedures included verification of data inconsistencies and outliers using the medical records. The following characteristics were obtained for all patients: age, body mass index (BMI), the presence or absence of previous abdominopelvic surgery, gravidity, parity, and the primary indication for surgery. Perioperative characteristics included concomitant procedures performed with the hysterectomy reflecting the complexity of the surgery, skin-to-skin operative time (defined as Foley catheter insertion to skin closure), uterine weight, conversion to laparotomy, estimated blood loss (EBL), length of hospital stay and intraoperative and postoperative complications up to 30 days post-discharge. Estimated blood loss was determined initially by canister collection as the differential between aspirated and irrigated fluids and necessitated agreement between the surgeon and anesthesiologist. Blood collection was later done with the Stryker Neptune system that also enabled quantification of very small amounts of blood loss that went undetectable using the original system. Thus, if no blood was detected by the canister collection method, EBL was recorded as $25 \mathrm{ml}$. Minor complications following discharge (such as urinary tract infection) were captured on the patient follow-up visit for robotic surgery. However, these were not available on all LAVH and LSH patients. Therefore, with regard to postoperative complications, only those patients requiring a visit to the emergency room or a readmission to the hospital were reported in this study.

\section{Statistical analyses}

Data analyses included all pair-wise comparisons of minimally invasive surgical methods, namely, RALH to each of the two other approaches (LAVH and LSH) as well as LAVH to LSH, using SAS software ver. 9.2.1 (SAS Institute, Cary, NC). Continuous variables were compared using two-sample $t$ tests. Discrete variables were analyzed using chi-squared tests or Fisher's exact test with continuity correction. In addition, multivariable regression analyses were used to identify patient and procedure characteristics that had a significant impact on operative time. To identify changes in operative time, blood loss and uterine weight that could be associated with surgical experience, these parameters were compared over the consecutive patient series for the RALH and LAVH procedures performed by the same surgeons (BG, HG). In all instances a $P$ value $<0.05$ was considered to be statistically significant.

\section{Results}

Comparisons of preoperative patient characteristics are shown in Table 1. The most frequent primary indication for benign hysterectomy among these patients was abnormal uterine bleeding in RALH (50\%) and LAVH (36\%) and fibroids in LSH (39\%). Endometriosis was the third most frequent indication, ranging from $8 \%$ in RALH patients to almost $21 \%$ in LSH cases. The age range of all patients was 23-78 years. Those who underwent LSH were younger on average than either RALH $(P=0.067)$ or LAVH $(P=0.006)$ patients by 1.5 and 2.5 years, respectively, not surprising given the desire for a cervix-preserving procedure. Differences in gravidity and parity followed similar trends, with RALH patients having had significantly fewer pregnancies and live births than either LAVH or LSH patients, with no differences between those in the LAVH and LSH groups. Means for BMI indicate that many patients were obese $(\mathrm{BMI} \geq 30$ ) or bordering on obesity. Differences in BMI by approach were small, and comparisons were not statistically significant. A large proportion of patients had undergone prior abdominopelvic surgery, 
Table 1 Preoperative characteristics by minimally invasive approach to hysterectomy

\begin{tabular}{|c|c|c|c|c|}
\hline Preoperative characteristics & RALH $(n=237)$ & LAVH $(n=265)$ & $\mathrm{LSH}(n=87)$ & $P$ value \\
\hline \multicolumn{5}{|l|}{ Primary indication for surgery, $n(\%)$} \\
\hline Benign pelvic mass & $1(0.4)$ & - & - & \\
\hline Abnormal uterine bleeding & $119(50.2)$ & $96(36.2)$ & $28(32.2)$ & \\
\hline Fibroids & $68(28.7)$ & $89(33.6)$ & $34(39.1)$ & \\
\hline Endometriosis & $19(8.0)$ & $27(10.2)$ & $18(20.7)$ & \\
\hline Pelvic pain & $11(4.6)$ & $14(5.3)$ & - & \\
\hline Abnormal PAP & - & $1(0.4)$ & - & \\
\hline Pelvic relaxation & $16(6.8)$ & $7(2.6)$ & - & \\
\hline Ovarian cyst & - & $2(0.8)$ & $3(3.4)$ & \\
\hline Prolapse (uterine/vaginal) & $3(1.3)$ & $17(6.4)$ & - & \\
\hline Other & - & $12(4.5)$ & $4(4.6)$ & \\
\hline \multicolumn{5}{|l|}{ Age (years) } \\
\hline Mean (SD) & $41.5(8.4)$ & $42.5(10.6)$ & $39.9(6.3)$ & $0.240^{\mathrm{a}}$ \\
\hline $95 \% \mathrm{CI}$ & $40.4-42.5$ & $41.2-43.8$ & $38.6-41.3$ & $\begin{array}{l}0.067^{b} \\
0.006^{c}\end{array}$ \\
\hline \multicolumn{5}{|l|}{ Gravidity, $n(\%)$} \\
\hline 0 & $24(10.1)$ & $8(3.0)$ & $3(3.4)$ & $0.003^{\mathrm{a}}$ \\
\hline 1 & $38(16.0)$ & $40(15.1)$ & $8(9.2)$ & $0.009^{\mathrm{b}}$ \\
\hline$\geq 2$ & $175(73.9)$ & $217(81.9)$ & $76(87.4)$ & $0.387^{\mathrm{c}}$ \\
\hline \multicolumn{5}{|l|}{ Parity, $n(\%)$} \\
\hline 0 & $28(11.8)$ & $13(4.9)$ & $4(4.6)$ & $0.007^{\mathrm{a}}$ \\
\hline 1 & $46(19.4)$ & $48(18.1)$ & $9(10.3)$ & $0.004^{\mathrm{b}}$ \\
\hline$\geq 2$ & $163(68.8)$ & $204(77.0)$ & $74(85.1)$ & $0.207^{\mathrm{c}}$ \\
\hline \multicolumn{5}{|l|}{ BMI } \\
\hline Mean (SD) & $30.3(7.5)$ & $29.9(6.7)$ & $31.2(7.7)$ & $0.530^{\mathrm{a}}$ \\
\hline $95 \% \mathrm{CI}$ & $29.4-31.2$ & $29.0-30.7$ & $29.6-32.8$ & $\begin{array}{l}0.343^{b} \\
0.132^{c}\end{array}$ \\
\hline Prior abdominal or pelvic surgery, $n(\%)$ & $197(83.1)$ & $194(73.2)$ & $69(79.3)$ & $\begin{array}{l}0.010^{\mathrm{a}} \\
0.529^{\mathrm{b}} \\
0.320^{\mathrm{c}}\end{array}$ \\
\hline
\end{tabular}

$R A L H$ Robotic-assisted laparoscopic hysterectomy, $L A V H$ laparoscopic-assisted vaginal hysterectomy, $L S H$ laparoscopic supracervical hysterectomy, $S D$ standard deviation, $C I$ confidence interval

a Comparison of RALH to LAVH

${ }^{b}$ Comparison of RALH to LSH

c Comparison of LAVH to LSH

with RALH patients exhibiting the highest percentage $(83.1 \%)$, which was significantly greater than that in patients who underwent LAVH $(73.2 \% ; P=0.01)$. Patients who underwent LSH did not differ from the other groups with respect to prior surgery. Comparisons of preoperative characteristics between LAVH patients of Drs. Giep versus the other nine surgeons show very similar baseline profiles, with almost identical mean ages (42.6 vs. 42.4 years, respectively) and very similar mean BMI (29.9 vs. $29.5 \mathrm{~kg} / \mathrm{m}^{2}$, respectively) and proportion with prior abdominopelvic surgeries ( 75.2 vs. $70.0 \%$ ). These findings provide reassurance that patients from the two groups of surgeons combined as one LAVH cohort were very similar.
Examination of intraoperative characteristics by approach indicates that concomitant procedures were more often performed with RALH (in 50.6\% of surgeries) than with either LAVH (in 26\%) or LSH (in 20.6\%; Table 2). Specifically, the proportion of patients who had surgical procedures for endometriosis or lysis of adhesions was significantly greater in the RALH group than in the LAVH $(P<0.001)$ or LSH $(P<0.001)$ groups. Similar findings were evident for pelvic reconstruction (RALH vs. LAVH $P=0.012$, RALH vs. LSH $P<0.001)$. Most pelvic reconstruction involved robotically assisted uterosacral fixation, anterior and posterior repair and pubovaginal sling with cystoscopy. These procedures would be expected to 
Table 2 Intraoperative and perioperative characteristics by minimally invasive approach to hysterectomy

\begin{tabular}{|c|c|c|c|c|}
\hline Intraoperative and perioperative characteristics & RALH $(n=237)$ & LAVH $(n=265)$ & LSH $(n=87)$ & $P$ value \\
\hline \multicolumn{5}{|l|}{ Concomitant procedures, $n(\%)$} \\
\hline Endometriosis/lysis of adhesions & $93(39.2)$ & $56(21.1)$ & $15(17.2)$ & $\begin{array}{l}<0.001^{\mathrm{a}} \\
<0.001^{\mathrm{b}} \\
0.520^{\mathrm{c}}\end{array}$ \\
\hline Pelvic reconstruction & $27(11.4)$ & $13(4.9)$ & $3(3.4)$ & $\begin{array}{l}0.012^{\mathrm{a}} \\
<0.001^{\mathrm{b}} \\
0.044^{\mathrm{c}}\end{array}$ \\
\hline \multicolumn{5}{|l|}{ Uterine weight $(\mathrm{g})$} \\
\hline Mean (SD) & $207.4(194.5)$ & $149.6(118.7)$ & $141.1(172.5)$ & $<0.001^{\mathrm{a}}$ \\
\hline $95 \% \mathrm{CI}$ & $182.5-232.6$ & $134.9-164.3$ & $105.2-177.1$ & $\begin{array}{l}0.005^{\mathrm{b}} \\
0.670^{\mathrm{c}}\end{array}$ \\
\hline \multicolumn{5}{|l|}{ Stratified uterine weight } \\
\hline$<250 \mathrm{~g}$ & $182(76.8)$ & $241(90.9)$ & $80(92.0)$ & $<0.001^{\mathrm{a}}$ \\
\hline$\geq 250 \mathrm{~g}$ & $55(23.2)$ & $24(9.1)$ & $7(8.0)$ & $\begin{array}{l}0.004^{\mathrm{b}} \\
0.867^{\mathrm{c}}\end{array}$ \\
\hline \multicolumn{5}{|l|}{ Skin to skin operative time (min) } \\
\hline Mean (SD) & $89.9(37.5)$ & $124.8(48.7)$ & $89.6(38.0)$ & $<0.001^{\mathrm{a}}$ \\
\hline $95 \% \mathrm{CI}$ & $84.9-94.5$ & 118.9-130.7 & $80.9-98.5$ & $\begin{array}{l}0.949^{\mathrm{b}} \\
<0.001^{\mathrm{c}}\end{array}$ \\
\hline \multicolumn{5}{|l|}{ Estimated blood loss (ml) } \\
\hline Mean (SD) & $59.0(75.7)$ & $167.9(146.0)$ & $65.7(60.7)$ & $<0.001^{\mathrm{a}}$ \\
\hline $95 \% \mathrm{CI}$ & $49.2-68.6$ & $150.2-185.6$ & $52.7-78.6$ & $\begin{array}{l}0.412^{\mathrm{b}} \\
<0.001^{\mathrm{c}}\end{array}$ \\
\hline \multicolumn{5}{|l|}{ Length of hospital stay (days) } \\
\hline Mean (SD) & $1.0(0.1)$ & $1.2(0.7)$ & $1.2(0.8)$ & $<0.001^{\mathrm{a}}$ \\
\hline $95 \% \mathrm{CI}$ & $1.0-1.03$ & $1.1-1.2$ & $1.0-1.4$ & $\begin{array}{l}0.022^{\mathrm{b}} \\
1.00^{\mathrm{c}}\end{array}$ \\
\hline Conversion, $n(\%)$ & $4(1.7)$ & $1(0.4)$ & None & $\begin{array}{l}0.194^{\mathrm{a}} \\
0.577^{\mathrm{b}} \\
1.00^{\mathrm{c}}\end{array}$ \\
\hline Intraoperative complications, $n(\%)$ & $1(0.4)$ & $1(0.4)$ & None & NA \\
\hline Postoperative complications, $n(\%)$ & $8(3.4)$ & $4(1.5)$ & $2(2.3)$ & $\begin{array}{l}0.243^{\mathrm{a}} \\
1.00^{\mathrm{b}} \\
0.640^{\mathrm{c}}\end{array}$ \\
\hline
\end{tabular}

$R A L H$ Robotic-assisted laparoscopic hysterectomy, $L A V H$ laparoscopic-assisted vaginal hysterectomy, $L S H$ laparoscopic supracervical hysterectomy, $S D$ standard deviation, $C I$ confidence interval, $N A$ Not available

a Comparison of RALH to LAVH

b Comparison of RALH to LSH

c Comparison of LAVH to LSH

contribute to an increase in operative time. Although the rates of pelvic reconstruction were low in both LAVH and LSH, they were statistically higher in LAVH (4.9\%) than LSH $(3.4 \% ; P=0.04)$. Uterine weights ranged from 24 to $1,233 \mathrm{~g}$. The mean uterine weight was similar in LAVH and LSH patients but differed significantly from that of RALH cases who had the largest uteri $(207.4 \pm 194.5$ (RALH) vs. $149.6 \pm 118.7 \quad(\mathrm{LAVH}) \mathrm{g}, \quad P<.001 ; \quad$ vs. $141.1 \pm 172.5$ (LSH) g, $P=0.005]$. The percentage of patients with uteri of at least $250 \mathrm{~g}$ was $23.2 \%$ in RALH compared to 9.1 and $8.0 \%$ in LAVH $(P<0.001)$ and LSH $(P=0.004)$, respectively. However, mean skin-to-skin operative time was $35 \mathrm{~min}$ longer for LAVH patients compared to both RALH and LSH patients for whom procedures took approximately $1.5 \mathrm{~h}$ on average (both comparisons, $P<0.001)$. Similarly, estimated blood loss was highest in LAVH, being over $100 \mathrm{ml}$ greater than in RALH and LSH (both comparisons, $P<0.001$ ). Length of 
hospital stay was short for all approaches, but significantly shorter for RALH patients than either LAVH $(P<0.001)$ or LSH $(P=0.022)$ patients.

The rates of conversion to an open procedure were low, ranging from zero to $1.7 \%$, and did not differ by surgical approach (Table 2). The conversions in the RALH group occurred early in the learning curve. Two conversions were within the first 25 cases and another two within the first 150 cases. These occurred in patients with large uteri where there was difficulty in accessing the blood vessels. Similarly, one conversion in the LAVH group occurred in a patient with a 16-week uterus where it was difficult to maintain hemostasis while proceeding laparoscopically. This patient was subsequently converted to an abdominal hysterectomy. Rates of intraoperative and postoperative complications within 30 days of surgery were low across the three surgical approaches: the overall rates totaled 3.8\% for RALH, $1.9 \%$ for LAVH and $2.3 \%$ for LSH, with the majority being minor in severity. No statistically significant differences were noted by surgical method. In both the RALH and LAVH cohorts, there was one intraoperative cystotomy repair with no further sequelae. There were also two patients with incisional infections and one with a bacterial infection in the RALH cohort; these were treated with antibiotics during their hospital stay. One patient in the robotic cohort and one in the LAVH cohort experienced a pelvic abscess that was subsequently drained and treated. There were no instances of cuff dehiscence requiring repair in any of the cohorts. There was one patient in the robotic group who was treated for cuff cellulitis, and two patients reported bleeding from the vaginal cuff in the RALH and LAVH cohorts. Both of these latter complications were resolved without the need for reintervention. During the perioperative period, one patient in the robotic group suffered a pulmonary embolism that was treated by anticoagulation with heparin and then enoxaparin (Lovenox). Atelectasis was noted in three patients, one from the LAVH cohort and two in the LSH cohort.

Comparisons of perioperative characteristics for the LAVH procedures performed by Drs. Giep versus the other surgeons show similar patient uterine weights $(153.4 \pm$ 124.5 vs. $147.0 \pm 114.4 \mathrm{~g}$, respectively), EBL (157.3 \pm 111.4 vs. $173.6 \pm 163.3 \mathrm{ml}$, respectively) and length of hospital stay (1.1 vs. 1.2 days, respectively). However, operative time was significantly shorter for procedures performed by Drs. Giep given their extensive experience with laparoscopic procedures $(101.5 \pm 39.7$ vs. $138.9 \pm$ 48.4; $P<.001)$.

Multivariable linear regression was used to identify those characteristics of the patients and the approach that were significant predictors of skin-to-skin operative time. The characteristics considered included patient age (dichotomized above or below the median age $=41$ years), obesity
(BMI $<30, \geq 30$ ), previous abdominal or pelvic surgery, uterine weight $(<250 \mathrm{~g}, \geq 250 \mathrm{~g})$, any concomitant procedure beyond hysterectomy with salpingo-oophorectomy and approach to surgery (RALH, LAVH, LSH). Younger age $(P<0.001)$, BMI $<30(P=0.02)$, uterine weight $<250 \mathrm{~g}$ $(P<0.001)$ and surgery other than $\operatorname{LAVH}(P<0.001)$ were all independently associated with shorter operative times (data not shown). On average, the operative times were longer for older patients versus younger ones $(21 \mathrm{~min})$, for obese patients versus non-obese patients ( $8 \mathrm{~min}$ ), for greater uterine weight patients versus those with a smaller uterus (24 min) and for those undergoing LAVH surgeries vs. those undergoing LSH and RALH (35 min).

Data points, representing the means of 25 sequential patients, were generated for skin-to-skin times, uterine weights and EBL over the entire case series for the RALH patients and for the comparable $100 \mathrm{LAVH}$ patients whose procedures were performed by the same surgeons (HG, BG). Comparisons of the first 25 cases to the last showed no statistically significant differences for any of the LAVH parameters, although uterine weights generally increased with longer surgical experience (data not shown). Similar findings were evident in RALH for uterine weight and EBL (comparison of first and last data points, $P=0.189$ and $P=0.875$, respectively). However, operative skin-to-skin time significantly decreased with surgical experience (comparison of first 25 to last 37 patients, $P=0.003$, Fig. 1). The first 25 RALH patients had a mean operative time of $106.4 \mathrm{~min}$, which is comparable to that of the LAVH procedures (101.5 min) and significantly different from the last patients' operative time of $76.5 \mathrm{~min}$.

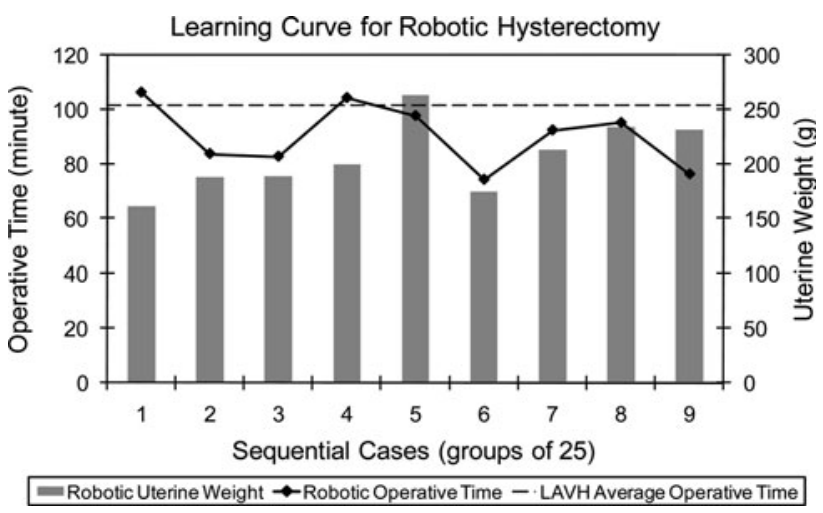

Fig. 1 Learning curve for robotic-assisted laparoscopic hysterectomy (RALH). Solid black line connects the mean skin-to-skin operative times at 25-case intervals for RALH $(P=0.003$ for mean of first 25 vs. last 37 patients). Dashed black line Mean skin-to-skin time for 100 comparable laparoscopic-assisted vaginal hysterectomy $(L A V H)$ patients (HG, BG only). There were no significant differences in operative time for the first $25 \mathrm{LAVH}$ cases vs. the last 25 cases, $P=0.59$. Gray bars Mean uterine weights corresponding to the each of the RALH 25 -case intervals $(P=0.189$ comparing first 25 vs. last 37 cases) 
Furthermore, as suggested by the regression analyses above, operative time varied with changes in uterine weight over time (Fig. 1).

\section{Discussion}

The results of this study show that while each of the three minimally invasive procedures had similar conversion and complication rates, there were distinct differences with regard to other perioperative outcomes. Although patients who underwent RALH had a higher rate of prior abdominopelvic surgeries than LAVH patients and more concomitant procedures performed and greater uterine weights than either LAVH or LSH patients, they experienced significantly lower operative times and EBL than LAVH patients and a significantly shorter length of hospital stay than either LAVH or LSH patients. Operative times were 35 min longer and EBL $100 \mathrm{ml}$ greater on average for LAVH patients than for those who underwent either the LSH or RALH procedures for whom these outcomes were comparable.

Multivariable analysis of the independent predictors of operative time confirmed the findings with regard to the impact of LAVH compared to LSH and RALH and further showed that older age, obesity, and large uteri ( $\geq 250 \mathrm{~g}$ ) in particular were also associated with longer surgical time. Despite the complexity of cases undertaken with RALH, including those with larger uteri, prior surgery and necessary concomitant procedures, the learning curve for operative time continued to improve over the case series, with mean times dropping from 106 to $76 \mathrm{~min}$ over the 23 months of surgery. The average operative time for the last $100 \mathrm{LAVH}$ cases performed by the same surgeons was $102 \mathrm{~min}$, with no noticeable decrease over the 20 months observed.

As previously mentioned, there are few published comparative studies on the outcomes of benign hysterectomy that include robotic procedures. Payne and Dauterive, who compared total laparoscopic hysterectomy in their last 100 patients to RALH in their first 100 patients, found longer average operative times for RALH but significantly shorter times in their last 25 robotic cases [7]. As in the our study, patients undergoing the total laparoscopic procedure had a greater EBL and a longer hospital stay than those undergoing the robotic hysterectomy. These researchers also found low complication rates that were similar in the two patient groups. A recently completed study also demonstrated significantly greater blood loss and longer hospital stay with standard laparoscopic hysterectomy compared to RALH [8]. While complication rates were similar in the two groups, conversion rates were significantly higher in the conventional laparoscopic cohort.
A similar comparative investigation showed that robotic hysterectomy patients had significantly shorter hospital stays but longer operative times than patients who underwent total laparoscopic hysterectomy [9]. To date, no comparative studies of RALH and LSH have been identified in the Medline literature database. Our study findings also indicate that outcomes in RALH, despite the increased complexity of the cases, were comparable to or better than those in LSH, suggesting that LSH should be considered for women primarily as a cervix-sparing surgery.

Studies comparing other minimally invasive procedures for hysterectomy are numerous and, as in our investigation, generally indicate better outcomes for patients undergoing LSH than LAVH. These better outcomes may be attributed to the fact that the LSH procedure avoids what can be a difficult bladder dissection that is required in LAVH. Milad and colleagues found that LSH patients had significantly shorter operative times and hospital stays, less blood loss and a lower complication rate than LAVH patients [11]. Similar to the results in our study, a large multi-center study investigation with matched groups of patients demonstrated significantly less blood loss and shorter operative times (by $30 \mathrm{~min}$ ) in the LSH patients compared to the LAVH patients and no difference in hospital stays [12]. Another large study of women undergoing a classic intrafascial supracervical hysterectomy reported lower complication rates and smaller amounts of EBL when compared to patients undergoing LAVH [13].

Several studies of hysterectomy for benign indications have also suggested that greater uterine weights or obesity are associated with longer operative times. Boggess et al. found that among preoperative patient characteristics, only uterine weight $>250 \mathrm{~g}$ was associated with increased operative time in RALH [14]. A comparative investigation of robotic and conventional laparoscopic hysterectomy reported significant independent effects of BMI and uterine weight on operative times [9]. Studies of robotic gynecologic procedures in patients unselected for pathology have also reported a relationship between greater uterine weight and longer duration of surgery or console time $[6,15]$. This relationship is usually attributed to the greater time needed for morcellation and/or removal of larger specimens. Investigations of other patients undergoing conventional laparoscopic hysterectomy generally confirm our findings regarding the impact of large uterine weight on operative time [16-18]. Several studies also have demonstrated a relationship between obesity and longer operative times in robotic or conventional laparoscopic hysterectomy $[15,19]$.

Other investigators have described the learning curve for robotic hysterectomy. They have documented shorter operative time with greater experience of the primary surgeon [5-7]. However, as surgeons become comfortable 
and competent in this technique, they also tend to take on more complex cases, a factor that could negatively impact operative time over a case series. Our study shows a visual example of the how operative time trends with the size of uteri in patients undergoing RALH over time.

The limitations to our study include the retrospective, observational nature of the design, introducing the potential for bias. However, with the exception of the small age difference in LSH patients, baseline characteristics of the three patient groups were fairly similar. RALH patients had a greater proportion of prior abdominopelvic surgeries and concomitant procedures and larger uterine weights than LAVH patients, but such differences would have only served to introduce a bias toward better LAVH outcomes. Our findings with regard to RALH versus LAVH do not support such a bias, although the differences found between RALH and LAVH may be conservative as a result. Misclassification errors, common in retrospective studies, also may have served to weaken tests of differences between surgical approaches. Furthermore, complication rates were low due to the exclusion of minor conditions, such as urinary tract infections, which may not have been captured during clinic visits after hospital discharge. These conditions were not routinely noted across all procedures and, therefore, could not be included.

\section{Conclusion}

Although the RALH patients in this study presented as the most complex cases in terms of prior pelvic surgery, concomitant procedures and uterine weight, they had better clinical outcomes with respect to operative time, EBL and length of hospital stay than the patients undergoing LAVH. On average, the operative time was 35 min longer and the EBL was $100 \mathrm{ml}$ greater for LAVH patients than for either RALH or LSH patients. Length of hospital stay was significantly greater for both LAVH and LSH patients (mean 1.2 days) than for RALH patients (mean 1.0 days). Other outcomes for LSH were similar to those for RALH. Conversions to laparotomy and intraoperative and postoperative complications were similar in the three patient groups. Our analysis of the learning curves suggest that operative time for RALH may continue to improve beyond the initial patient series despite the increased complexity of cases undergoing this procedure. The results of this study lend support to the small but growing body of literature describing the benefits of minimally invasive roboticassisted laparoscopic over conventional laparoscopicassisted vaginal hysterectomy. Our findings also demonstrate that outcomes with robotic surgery are as good as or even better than those for laparoscopic supracervical hysterectomy, suggesting that decisions regarding choice of the latter approach be based primarily on the desire for a cervix-sparing procedure.

Acknowledgments The authors wish to thank Usha Kreaden, MSc of Intuitive Surgical for statistical support during the completion of this manuscript.

Conflicts of interest Drs. Bang and Hoang Giep are members of Intuitive Surgical's Speakers' Bureau, Proctor Cases, and have actively been running "Epicenter" programs at their institution. Dr. Hubert is an epidemiologist who consults with Intuitive Surgical.

Open Access This article is distributed under the terms of the Creative Commons Attribution Noncommercial License which permits any noncommercial use, distribution, and reproduction in any medium, provided the original author(s) and source are credited.

\section{References}

1. Whiteman MK, Hillis SD, Jamieson DJ et al (2008) Inpatient hysterectomy surveillance in the United States, 2000-2004. Am J Obstet Gynecol 198:34.e1-34.e7

2. Jacoby VL, Autry A, Jacobson G, Domush R, Nakagawa S, Jacoby A (2009) Nationwide use of laparoscopic hysterectomy compared with abdominal and vaginal approaches. Obstet Gynecol 114:1041-1048

3. Johnson N, Barlow D, Lethaby A, Tavender E, Curr L, Garry R (2005) Methods of hysterectomy: systematic review and metaanalysis of randomized controlled trials. Br Med J 330:1478-1485

4. Falcone T, Paraiso MFR, Mascha E (1999) Prospective randomized clinical trial of laparoscopically assisted vaginal hysterectomy versus total abdominal hysterectomy. Am J Obstet Gynecol 180:955-962

5. Pitter MC, Anderson P, Blissett A, Pemberton N (2008) Roboticassisted gynaecological surgery-establishing training criteria; minimizing operative time and blood loss. Int J Med Robotics Comput Assist Surg 4:114-120

6. Lenihan FR Jr, Kovanda C, Seshadri-Kreaden U (2008) What is the learning curve for robotic assisted gynecologic surgery? J Minim Invasive Gynecol 15:589-594

7. Payne TN, Dauterive FR (2008) A comparison of total laparoscopic hysterectomy to robotically assisted hysterectomy: surgical outcomes in a community practice. J Minim Invasive Gynecol $15: 286-291$

8. Landeen LB, Bell MC, Hubert HB, Bennis LY, Knutsen-Larson SS, Seshadri-Kreaden U (2010) Clinical and cost comparisons: robot-assisted versus abdominal, standard laparoscopic and vaginal hysterectomy. Gray $\mathrm{J}$ (submitted for publication)

9. Shashoua AR, Gill D, Locher SR (2009) Robotic-assisted total laparoscopic hysterectomy versus conventional total laparoscopic hysterectomy. J Soc Laparoendosc Surg 13:364-369

10. Lalonde CJ, Daniell JF (1996) Early outcomes of laparoscopicassisted vaginal hysterectomy versus laparoscopic supracervical hysterectomy. J Am Assoc Gynecol Laparosc 3:251-256

11. Milad MP, Morrison K, Sokol A, Miller D, Kirkpatrick L (2001) A comparison of laparoscopic supracervical hysterectomy vs. laparoscopically assisted vaginal hysterectomy. Surg Endosc 15:286-288

12. El-Mowafi D, Facharzt WM, Lall C, Wenger JM (2004) Laparoscopic supracervical hysterectomy versus laparoscopicassisted vaginal hysterectomy. J Am Assoc Gynecol Laparosc 11:175-180 
13. Kim DH, Bae DH, Hur M, Kim SH (1998) Comparison of classic intrafascial supracervical hysterectomy with total laparoscopic and laparoscopic-assisted vaginal hysterectomy. J Am Assoc Gynecol Laparosc 5:253-260

14. Boggess JF, Gehrig PA, Cantrell L et al (2009) Perioperative outcomes of robotically assisted hysterectomy for benign cases with complex pathology. Obstet Gynecol 114:585-593

15. Payne TN, Dauterive FR (2010) Robotically assisted hysterectomy: 100 cases after the learning curve. J Robotic Surg 4:11-17

16. Wattiez A, Soriano D, Fiaccavento A et al (2002) Total laparoscopic hysterectomy for very enlarged uteri. J Am Assoc Gynecol Laparosc 9:125-130
17. Seracchioli R, Venturoli S, Colombo FM et al (2003) GnRH agonist treatment before total laparoscopic hysterectomy for large uteri. J Am Assoc Gynecol Laparosc 10:316-319

18. Bonilla DJ, Mains L, Whitaker R, Crawford B, Finan M, Magnus M (2007) Uterine weight as a predictor of morbidity after a benign abdominal and total laparoscopic hysterectomy. J Reprod Med 52:490-498

19. Chopin N, Malaret JM, Lafay-Pillet MC, Fotso A, Foulot H, Chapron C (2009) Total laparoscopic hysterectomy for benign uterine pathologies: obesity does not increase the risk of complications. Hum Reprod 24:3057-3062 